Ann. Biol. anim. Bioch. Biophys., I974, 14 (ז), 53-66.

\title{
ASPECTS ORIGINAUX \\ DE LA GLANDE INTERSTITIELLE OVARIENNE GHEZ LE HÉRISSON : MORPHOLOGIE, HISTOGENESE, HYPERPLASIE, ENZYMES DE LA STÉROÏDOGENESE
}

\author{
A. GOUGEON ${ }^{(1)}$ \\ Laboratoire de Physiologie comparée \\ Faculté des Sciences, 86022 Poitiers, \\ Centre d'Études biologiques des Animaux sauvages du C. N. R. S., \\ 79360 Beauvoir sur Niort
}

\section{RÉSUMÉ}

Chez le Hérisson européen, la glande interstitielle de l'ovaire se présente sous la forme d'un nodule localisé au niveau du hile : c'est le nodule interstitiel hilaire (n. i. h.).

Situé à proximité des tubules du rete ovarii, il n'apparaît chez la jeune femelle qu'à l'âge de 3 mois (6 à 9 mois avant la puberté). LH et FSH permettent son induction précoce.

Il provient de l'évolution des cellules de certains tubules du rete ovarii ; son volume s'accroît progressivement par apport et différenciation de cellules conjonctives fusiformes issues de la médulla ovarienne.

L'analyse histoenzymologique montre que l'équipement $d u \mathrm{n}$. $\mathrm{i}$. h. en enzymes de la stéroïdogenèse est proche de celui du corps jaune. La $\Delta_{5} * 3 \beta$-HSD est l'enzyme la plus active, les I $7 \beta$ et $6 \beta$-HSD sont aussi mises en évidence.

La diminution du volume des cellules interstitielles pendant la période d'activité sexuelle refléterait l'existence d'une synthèse hormonale suivie de sécrétion.

Le $n$. i. h. est sujet à l'hyperplasie chez les animaux âgés ( $\geqslant$ à 4 ans). Le nodule atteint alors des dimensions importantes ( 2 à $5 \mathrm{~mm}$ de diamètre). Les cellules interstitielles hypertrophiées constituent le seul type cellulaire, il n'y a pas de mitose.

\section{INTRODUCTION}

L'ovaire du Hérisson (Erinaceus europeus) a été l'objet de quelques travaux effectués principalement par Limon (I903), Athias (I923), VELLLOSO DE Pinho (1923), DeAnesty (I934), Girod et al. (I967), Wai,in et al. (I968), Bat,Boni (I969), MarchaiDACHEUX (I970).

(1) Nouvelle adresse : Obstétrique et Gynécologie, Maternité de l'Hôpital Antoine Béclère, I57, rue de la Porte de Trivaux, 92140 Clamart. 
La glande interstitielle n'a jamais été étudiée de façon approfondie, seuls quelques auteurs signalent brièvement son existence en lui attribuant une origine thécale.

En I970, MARCHAL-DACHEUX met en évidence une formation composée de cellules interstitielles située au hile de l'ovaire; elle lui donne le nom de " nodule interstitiel hilaire" (n. i. h).

Par sa localisation et par sa morphologie bien définie, ce n. i. h. présente des caractères originaux. Nous avons cherché à en préciser la différenciation et l'évolution avec l'âge, ainsi que la fonction.

\section{I. - MATÉRIEI FT MÉTHODES}

\section{A. - Animaux}

Ils proviennent pour une part de l' "élevage \& du Centre d'Études biologiques des Animaux sauvages du C. N. R. S. (Forêt de Chizé) où certains sujets se reproduisent. Les autres hérissons ont été capturés dans la région de Poitiers.

Après anesthésie légère, les animaux sont décapités. Un ovaire et le tractus génital sont fixés au Bouin ou au Bouin Hollande, sublimé ou non. L'autre gonade est congelée rapidement, enveIoppée dans du "Parafilm " et stockée à $-18^{\circ} \mathrm{C}$.

\section{B. - Détermination quantitative des types cellulaires}

Nous avons utilisé pour cette étude un "Visopan " dont l'écran est divisé en carrés de $5 \mathrm{~mm}$ de côté.

Pendant l'ancestrus hivernal, le $n$. i. h. contient deux catégories de cellules interstitielles; on compte les points d'intersection se superposant à chacun des types cellulaires.

Le rapport des points est égal au rapport des surfaces, ce dernier étant identique au rapport des volumes (SOLARI, 1973); nous pouvons écrire: $\frac{\text { IOO } \times x}{y}=$ pourcentage du volume du n. i. h. occupé par des cellules du type $\mathbf{I}$.

$x$ : nombre de points correspondant aux cellules de type $\mathrm{I}$

$y$ : nombre de points correspondant à la surface du n.i.h.

Le nombre de cellules par unité de surface est inversement proportionnel à leur taille; il constitue un bon critère d'appréciation de l'état dans lequel se trouvent ces cellules à un moment donné.

\section{C. -- Analyses histologique et histoenzymologique}

\section{a) Analyse histologique.}

Les différents organes inclus dans la paraffine sont coupés à 7 ou Io $\mu$. Les colorations utilisées sont l'hémalun éosine, l'hémalun picroindigocarmin et le bleu Masson.

\section{b) Analyse histoenzymologique.}

Nous avons étudié l'équipement du $\mathrm{n}$. i. h. en enzymes de la stéroïdogenèse.

La technique utilisée découle de la méthode de WATTENBERG (1958). La composition du milieu étant inspirée des données fournies par Levy et al. (1959) pour les enzymes de la stéroïdogenèse et de celles de NACHLAS et al. (I957) pour la succinodéshydrogénase.

L'enrobage de l'ovaire dans de la gélatine à $5 \mathrm{p}$. ıoo facilite la réalisation des coupes effectuées à l'aide d'un cryostat $\left(t=-25^{\circ} \mathrm{C}\right)$, elles sont collées sur une lamelle, puis mises à sécher $5 \mathrm{mn}$ à l'air libre.

Chez le Hérisson, un repérage du n. i. h. par coloration (hémalun P. I. C.) de la coupe est nécessaire. gènes.

Les coupes sont rincées $5 \mathrm{mn}$ dans du tampon phosphate afin de déplacer les stérö̈des endo- 
Un prétraitement d'usage par l'acétone à $4^{\circ} \mathrm{C}$ a été tenté. Il s'est révélé déprimer l'activité enzymatique et n'a pas été généralisé.

Le milieu d'incubation est le suivant :

Nitro Bleu de tétrazolium (N. B. T.) . . . . .

Coenzyme $\mathrm{NAD}^{+} \ldots \ldots \ldots \ldots \ldots \ldots \ldots \ldots \ldots \ldots$

Tampon phosphate (selon Sorensen) .......

Substrat (voir tabl. I) :

Succinate de sodium..............

Stéroïde . . . . . . . . . . . . . . .
$0,5 \mathrm{ml} \mathrm{d}$ 'une solut. à I $\mathrm{mg} / \mathrm{ml} \mathrm{H}_{2} \mathrm{O}$ distillée $0,5 \mathrm{ml}$ d'une solut. à $3 \mathrm{mg} / \mathrm{ml} \mathrm{H}_{2} \mathrm{O}$ distillée $5 \mathrm{ml}$ d'une solut. à $\mathrm{pH} 7,4$

$0,5 \mathrm{ml}$ d'une solut. $0,2 \mathrm{M}$

$0, \mathbf{I} \mathrm{ml}$ d'une solut. à $2 \mathrm{mg} / \mathrm{ml}$ de $\mathrm{N}$. N. diméthyl formamide.

\section{TABLEAU I}

Histoenzymologie.

Enzymes testées et substrats utilisés

$3 \alpha$-Hydroxystéroüde-déshydrogénase :

$5 \alpha$-androstane- $3 \alpha$-ol-17-one (androstérone).

$5 \beta$-androstane- $3 \alpha$-ol-17-one (étiocholanolone).

$\Delta_{5}-3 \beta$-hydroxystéroîde-déshydrogénase :

Pregn-5-ène-3 $\beta$-ol-20-one (prégnènolone).

Pregn-5-ène-3 $\beta, 17 \alpha$-diol (17 $\alpha$-hydroxy prégnènolone).

Androst-5-ène-3 $\beta$-ol-17-one (déhydroépiandrostérone).

Androst-5-ène-3 $\beta, 17 \beta$-diol (androstènediol).

$6 \beta$-Hydroxystéroüde-déshydrogénase:

Pregn-4-ène-6 $\beta$-ol-3, 20-dione (6 $\beta$-hydroxyprogestérone).

$11 \alpha$-hydroxystéroïde-déshydrogénase:

Pregn-4-ène-11 $\alpha$-ol-3, 20-dione (11 $\alpha$-hydroxyprogestérone).

\section{$11 \beta$-hydroxystéroz̈de-déshydrogénase:}

Pregn-4-ène-11 $\beta, 17 \alpha, 21$-triol-3, 20-dione (cortisol).

Androst-4-ène-11 $\beta$-ol-3, 17-dione (11 $\beta$-hydroxyandrostènedione).

$16 \propto$-hydroxystéroïde-déshydrogénase :

Estra-1, 3, 5 (10)-triène-3, $16 \alpha$-diol-17-one (16 $\alpha$-hydroxyœstrone).

Estra-1, 3, 5 (10)-triène-3,17 $\beta, 16 \alpha$-triol (œestriol).

$16 \beta$-hydroxystéroïde-déshydrogênase:

Estra-1, 3, 5 (10)-triène-3, $16 \beta$-diol-17-one diacétate (16 $\beta$-hydroxyœstrone diacétate).

Estra-1, 3, 5 (10)-triène-3, $16 \beta, 17-\beta$-triol (16-épioestriol).

$17 \alpha$-hydroxystéroüde-déshydrogénase :

Estra-1, 3, 5 (10)-triène-3, $17 \alpha$-diol (cstradiol-17 $\alpha$ ).

$17 \beta$-hydroxystéroüde-déshydrogénase:

Estra-1, 3, 5 (10)-triène-3, $17 \beta$-diol (cestradiol-17 $\beta$ ).

Androst-4-ène-17 $\beta$-ol-3-one (testostérone).

$20 \alpha$-hydroxystéroïde-déshydrogénase :

Pregn-4-ène-20 $\alpha$-ol-3-one (20 $\alpha$-dihydroprogestérone).

$5 \alpha$-pregnane-3 $\alpha, 20 \alpha$-diol (pregnanediol).

$20 \beta$-hydroxystéroïde-déshydrogénase:

Pregn-4-ène-20 $\beta$-ol-3-one (20 $\beta$-dihydroprogestérone). 
La concentration finale en stéroïdes du milieu est de l'ordre de $30 \mu \mathrm{g} / \mathrm{ml}$; cette faible quantité de substrat présente l'avantage de n'être pas trop éloignée des concentrations physiologiques et de diminuer la quantité de diméthyl formamide utilisée.

L'utilisation de plusieurs substrats par enzyme est justifiée par leur spécificité, un substrat n'étant pas suffisant pour s'assurer de la présence ou de l'absence d'une enzyme donnée. C'est ainsi que chez la Taupe (GougEoN, I972) l'utilisation de la 20 -dihydroprogestérone permet la mise en évidence d'une forte activité $20 \alpha$-HSD, le pregnandiol étant inefficace.

L'incubation dure une heure, la coupe est fixée $15 \mathrm{mn}$ dans l'alcool formol, lavée à l'eau distillée puis montée au glycérogel.

\section{II. - RÉSULTATS}

\section{A. - Situation et morphologie du n. i. h.}

Le n. i. h. est toujours situé en position hilaire, à proximité immédiate des tubules du rete ovarii (fig. 2 , planche I) ; son diamètre augmente avec l'âge, il passe de roo $\mu$ chez les animaux les plus jeunes à $600-700 \mu$ chez les adultes. Plusieurs nodules interstitiels de petite taille ( 50 à roo $\mu$ de diamètre) peuvent voisiner, puis se confondre en un n. i. h. plus gros.

Les cellules interstitielles peuvent se présenter sous trois aspects :

Type 1: cellules inactives à petit noyau rond ( $3 \mu$ de diamètre), de petite taille ( $5 \mu$ de diamètre environ), elles sont surtout regroupées en ilots (fig. I, pl. II).

Type 2: cellules polyédriques à noyau globuleux $(5 \mu$ de diamètre $)$ renfermant plusieurs nucléoles. De grande taille pendant l'anœstrus hivernal $(5 \mu \times$ ro $\mu)$ (fig. 2, pl. II), elles retrouvent une taille moyenne au printemps $(5 \mu \times 7 \mu)$; elles sont hypertrophiées (Io $\mu \times \mathbf{1} 6 \mu$ ) au niveau des nodules hyperplasiés (fig. $3, \mathrm{pl}$. II).

Type 3: de mêmes caractéristiques générales que les cellules du type 2 hypertrophiées, leur cytoplasme est fortement éosinophile; elles sont vraisemblablement en dégénérescence (fig. 3, pl. II).

Les petites cellules conjonctives fusiformes sont nombreuses au sein du n, i. h. des jeunes femelles impubères. Elles semblent se différencier en cellules interstitielles de type I.

Les capillaires sanguins y abondent; en outre, de nombreux vaisseaux hilaires cotoient ce nodule, par ailleurs enclavé dans un conjonctif médullo-hilaire lâche (fig. I, pl. I).

Il n'y a pas de cristaux de Reincke au niveau de ces cellules; elles ne sont donc pas analogues aux cellules du hile de l'ovaire rencontrées chez la Femme et la Truie.

\section{B. - Age et déterminisme de l'apparition du n. i.h. chez le jeune Hérisson femelle}

MaRchal-Dacheux (I970) émettait à propos du n. i. h., l'hypothèse suivante : " Nous pourrions être en présence de cellules sympathicotropes, analogues à celles des Primates et des Porcelets. Absentes chez le jeune Hérisson de trois semaines, elles apparaîtraient dans l'ovaire à la puberté. "

Afin de préciser le moment d'apparition de ce tissu, nous avons observé de la naissance jusqu'à l'âge de 6 mois, les ovaires de 30 femelles impubères. 
- A la fin de l'ovogenèse, vers la $3^{\mathrm{e}}$ semaine post natale, le $\mathrm{n}$. i. h. est absent.

- Vers 3 mois, quelques follicules possèdent un antrum, le n. i. h. est alors présent.

D'abord de taille modeste (30 à $40 \mu$ de diamètre), il croît rapidement, tandis que les cellules, de petite taille, mais dotées d'un noyau globuleux, évoluent rapidement vers la forme polyédrique.

Le nodule interstitiel semble donc se différencier, chez le jeune Hérisson femelle, vers l'âge de trois mois, au moment où apparaissent les follicules à antrum.

\section{C. - Essai d'induction du n. i. h. par les gonadotropines LH et FSH}

Nous avons cherché à induire précocement la formation du n. i. h. par l'administration de gonadotropines, la glande interstitielle ovarienne de divers Mammifères étant particulièrement sensible à ces hormones (PONSE, I965; AsChHEIM, I968; Armstrong et al., I969).

Des Hérissons âgés de 2,5 mois au maximum, ont reçu des injections de $\mathrm{LH}$ bovine (NIH-LH-B 7) ou de FSH porcine (NIH-FSH-P I).

- Le protocole expérimental est résumé sur le tableau 2.

\section{TABLEAU 2}

Traitement de jeunes Hérissons femelles par les gonadotropines

(NIH-LH-B 7 et NIH-FSH-P I)

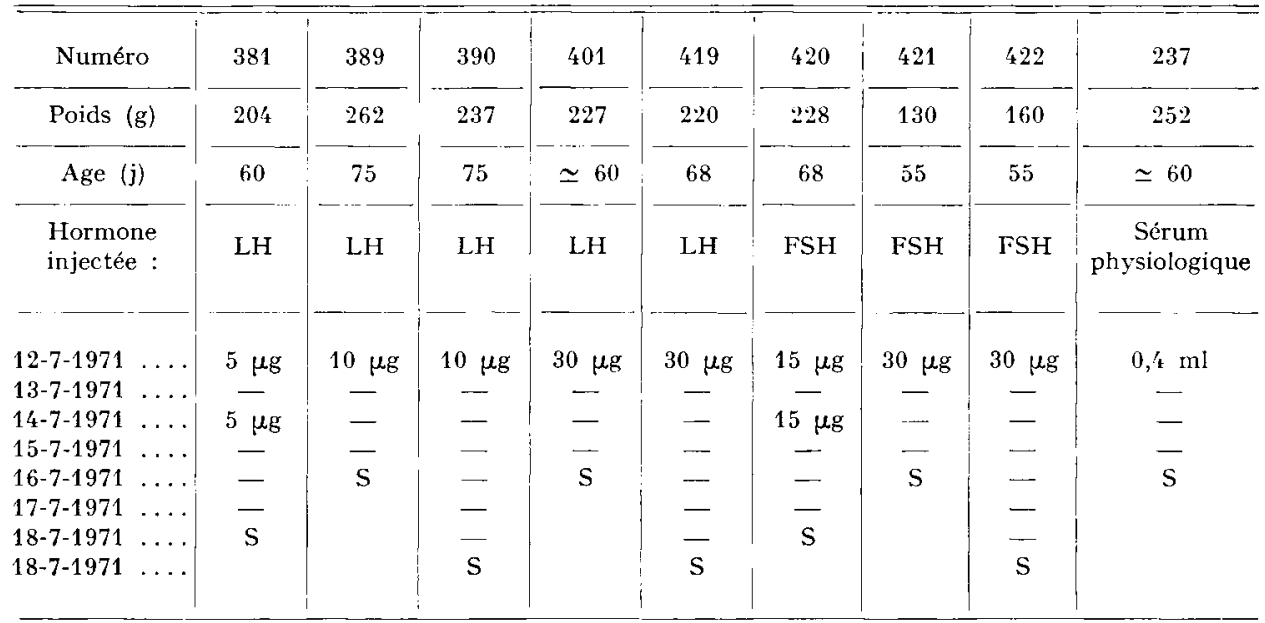

Les quantités d'hormone injectée sont exprimées en $\mu \mathrm{g}$ pour $100 \mathrm{~g}$ de poids corporel.

$\mathrm{S}$ : sacrifice.

- Plusieurs points ont retenu notre attention :

- Il n'y a pas de différence significative entre l'aspect histologique des ovaires d'animaux traités soit par LH, soit par FSH.

- Le nombre des follicules à antrum est important; ils sont généralement atrétiques et ne montrent pas de lutéinisation des thèques ou de la granulosa. 
Rappelons que l'ovaire de l'animal témoin ne comporte pas encore de follicules à antrum.

- Les Hérissons traités présentent un ou plusieurs $n$. i. h. de petite taille (50 à Ioo $\mu$ de diamètre). Tous sont situés à proximité des tubules du rete ovarii. Les cellules ont un gros noyau ( $5 \mu$ de diamètre), leur cytoplasme est spumeux. Aucune mitose n'y est visible.

\section{D. - Histogenèse du n. i. h.}

Chez le Hérisson, la position du n. i. h. et l'étude des coupes, après stimulation hormonale ou non, montrent que toute participation thécale à l'élaboration du n. i. h. doit être exclue. En fait, c'est au niveau du hile que certaines cellules se différencient en cellules interstitielles.

Il ne semble pas que le n. i. h. du Hérisson soit comparable aux formations, d'origine surrénalienne, observées dans l'ovaire de nombreux Mammifères ("Adreno Cortical Like cells "). Cette conclusion est confirmée par quelques observations : âge tardif d'apparition du n. i. h., comparaisons histologique et histoenzymologique du tissu interstitiel et des différentes zones du cortex surrénal.

Chez cet Insectivore, la glande interstitielle est toujours située à proximité des tubules du rete ovarii (vestiges de la partie crâniale du corps et du canal de Wolff).

Quelques images montrent la différenciation de cellules adjacentes à certains tubules du rete ovarii, d'autres, une évolution de l'épithélium de ces mêmes formations en cellules de type interstitiel (fig. 4, p1. I).

La présence de nodules multiunitaires et la similitude morphologique de nombre d'entre eux avec les cordons du rete (fig. 3, p. I) semblent confirmer cette évolution, les fibres conjonctives présentes dans le $\mathbf{n}$. i.h. pouvant être résiduelles et provenir de la destruction de la capsule des tubules transformés.

En conclusion, nous pensons que le $\mathrm{n}$. i. h. est issu de la différenciation de cellules de certains tubules du rete ovarii et des cellules conjonctives situées à leur contact.

\section{E. - Évolution du n. i. h. au cours de la vie sexuelle}

Nous avons observé les ovaires de 46 femelles adultes, à tous les stades du cycle, 6 d'entre elles étaient gestantes.

Le volume du n. i. h. s'accroît avec l'âge de l'animal, non par multiplication cellulaire, mais par apport et différenciation d'éléments extérieurs vraisemblablement issus de la médulla ovarienne (cellules conjonctives fusiformes).

\section{Des variations cycliques affectent la taille des cellules interstitielles.}

A la fin de l'automne et pendant l'hiver, il existe 2 types de n. i. h. suivant l'âge des animaux :

- Chez les jeunes femelles nées au printemps le $n$. i. h. est petit : roo $\mu$ de diamètre en moyenne. Il renferme des cellules interstitielles du type 2 et de nombreuses cellules conjonctives, jusqu'à $60 \mathrm{p}$. Ioo du nombre total de cellules.

- Chez les femelles adultes, le n. i. h. est plus gros : $600 \mu$ de diamètre en moyenne. Il est composé : 
- de cellules interstitielles de type I regroupées en îlots,

- de grosses cellules de type 2 .

Les populations de chacun de ces types sont sensiblement égales. On y trouve aussi des cellules conjonctives, mais il est très difficile d'apprécier leur importance numérique.

Au début du printemps, approximativement au moment de l'œstrus, il n'existe plus de différence liée à l'âge, les jeunes femelles devenant pubères. De plus, toutes les cellules interstitielles sont de type 2 et de taille moyenne $(5 \mu \times 7 \mu)$. (Cette évolution est résumée sur le schéma $I$ et le tableau 3).

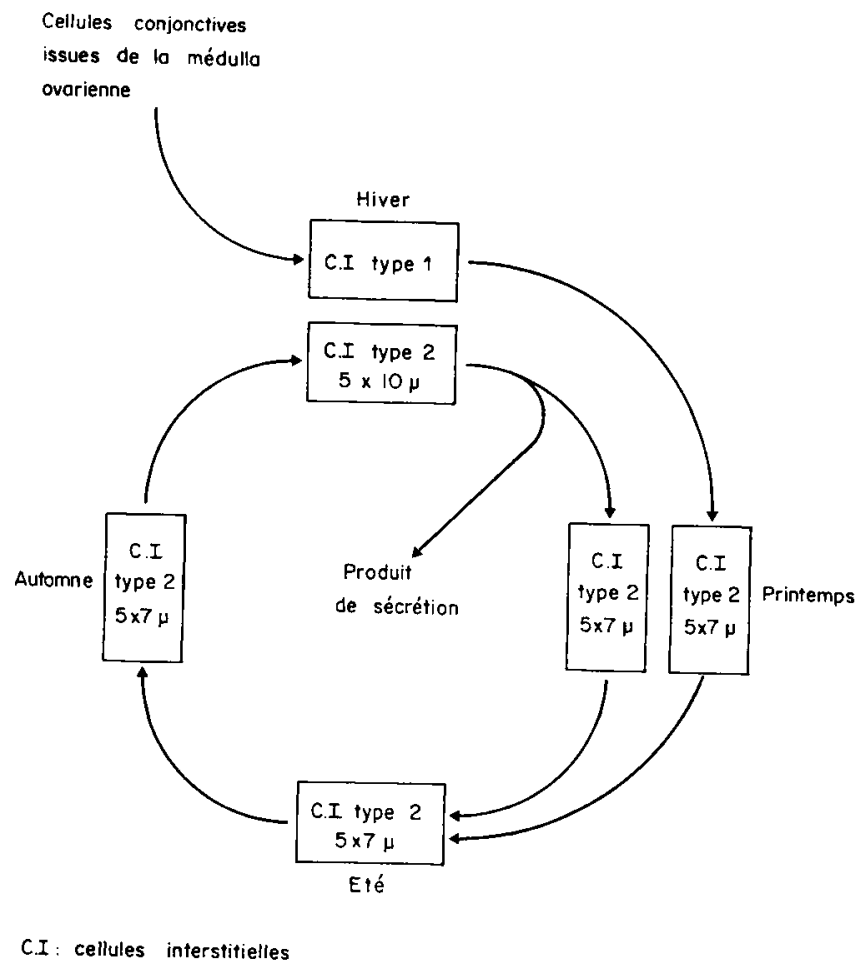

SснÉma I. - Évolution cyclique des cellules interstitielles du $n$. $i$. h. ovarien chez le Hérisson

TABLEAU 3

\begin{tabular}{|c|c|c|c|}
\hline & \multicolumn{2}{|c|}{ Cellules interstitielles } & \multirow{2}{*}{$\begin{array}{l}\text { Nombre de cellules } \\
\text { par unité de surface }\end{array}$} \\
\hline & $\begin{array}{c}\text { inactives, type } 1 \\
(\%)\end{array}$ & $\begin{array}{c}\text { actives, type } 2 \\
(\%)\end{array}$ & \\
\hline Ancestrus hivernal & $\begin{array}{c}25 \ll 30 \\
\text { de la } \mathrm{S} \text { du n. i. h. }\end{array}$ & $\begin{array}{c}70 \ll 75 \\
\text { de la } S \text { du n. i. h. }\end{array}$ & $\begin{array}{llllr}\text { Inactives } & : & 80 & \text { à } & 100 \\
\text { Actives } & : & 25 & \text { à } & 35\end{array}$ \\
\hline Gestation & $\simeq 0$ & $\simeq 100$ & Actives $: 40$ à 50 \\
\hline Activité sexuelle & $\simeq 0$ & $\simeq 100$ & Actives : 4 ' à 60 \\
\hline
\end{tabular}


Activité des hydroxystéroide-déshydrogénases (HSD) e - : activité nulle; $\quad(+)$ : très faib

\begin{tabular}{|c|c|c|c|c|c|c|c|c|c|}
\hline & \multirow{2}{*}{$\begin{array}{l}\text { Succi- } \\
\text { nate } \\
\text { de } \mathrm{Na}\end{array}$} & \multicolumn{2}{|c|}{$3 \alpha$-HSD } & \multicolumn{4}{|c|}{$\Delta_{5}-3 \beta$-HSD } & \multirow{2}{*}{$\frac{6 \beta-\mathrm{HSD}}{\frac{\substack{6 \beta-\mathrm{OH} \\
\mathrm{Pg}}}{-}}$} & \multirow{2}{*}{$\begin{array}{c}11 \alpha-\mathrm{HS} \\
\begin{array}{c}11 \alpha-\mathrm{OH} \\
\mathrm{Pg}\end{array}\end{array}$} \\
\hline & & $\begin{array}{l}\text { Andro- } \\
\text { stérone }\end{array}$ & $\begin{array}{l}\text { Étiocho- } \\
\text { lanolone }\end{array}$ & Pn & $\begin{array}{c}17 \alpha-\mathrm{OH}- \\
\mathrm{Pn}\end{array}$ & DHEA & $\begin{array}{l}\text { Andro- } \\
\text { stènediol }\end{array}$ & & \\
\hline Anœestrus & ++ & - & $(+)$ & +++ & + & +++ & +++ & & - \\
\hline Activité sex. & $+t+$ & $(+)$ & $(+)$ & ++ & + & +++ & +++ & + & 一 \\
\hline Gestation & $++t$ & $(+)$ & $(+)$ & ++ & + & +++ & +++ & + & - \\
\hline Hyperplasies & ++ & + & $(+)$ & +++ & + & +++ & +++ & & - \\
\hline
\end{tabular}

Coupes témoins : incubées en l'absence de substrat.
Abreviations :
Pn : Prégnénolone.
$\mathrm{E}_{1}$ : Estrone.
DHEA : Déhydroépiandrostéone.
$\mathrm{E}_{2}$ : CEstradiol
Pg : Progestérone.
$\mathrm{E}_{3}$ : Estriol.

\begin{tabular}{|c|c|c|c|c|c|c|c|c|c|}
\hline \multicolumn{10}{|c|}{$\begin{array}{r}\text { Équipement stéroïdogène du n. i. h., du corps jaune et du cort } \\
\text { Activité :- : nulle } \quad(+): \text { très faibl }\end{array}$} \\
\hline & \multirow{2}{*}{$\begin{array}{l}\text { Succi- } \\
\text { nate } \\
\text { de Na }\end{array}$} & \multicolumn{2}{|c|}{$3 \alpha$-HSD } & \multicolumn{4}{|c|}{$\Delta_{5}-3 \beta-$ HSD } & \multirow{2}{*}{$\frac{6 \beta-\mathrm{HSD}}{\frac{{ }_{\mathrm{Pg}} \mathrm{P}-\mathrm{OH}}{\mathrm{Pg}}}$} & \multirow{2}{*}{$\begin{array}{c}11 \alpha \text {-HSI } \\
\begin{array}{c}\alpha-\mathrm{OH} \\
\mathrm{Pg}\end{array}\end{array}$} \\
\hline & & $\begin{array}{l}\text { Andro- } \\
\text { stérone }\end{array}$ & $\begin{array}{l}\text { Etiocho- } \\
\text { lanolone }\end{array}$ & $\mathrm{Pn}$ & $\begin{array}{c}17 \alpha-\mathrm{OH}- \\
\mathrm{Pn}\end{array}$ & DHEA & $\begin{array}{l}\text { Andro- } \\
\text { stènediol }\end{array}$ & & \\
\hline N. i. h. & +++ & $(+)$ & $(+)$ & ++ & + & +++ & +++ & + & - \\
\hline Corps jaune & +++ & $(+)$ & - & ++ & $(+)$ & +++ & +++ & + & $(+)$ \\
\hline $\begin{array}{c}\text { Cortex } \\
\text { surrénalien }\end{array}$ & +++ & - & 一 & +++ & $(+)$ & +++ & +++ & ++ & $(+)$ \\
\hline
\end{tabular}

Coupes témoins incubées sans substrat.

Abréviations : $\quad$ Pn : Prégnénolone.

DHEA : Déhydroépiandrostérone.

$\mathrm{E}_{1}$ : Estrone.

Pg : Progestérone.

$\mathrm{E}_{2}:$ :Estradiol.

$\mathrm{E}_{3}$ : Estriol. 
U 4

succino oxydase au niveau du n. $i$. h. chez le Hérisson

: faible; $\quad++$ : moyenne; +++ : forte

\begin{tabular}{|c|c|c|c|c|c|c|c|c|c|c|c|}
\hline \multicolumn{2}{|c|}{$11 \beta$-HSD } & \multicolumn{2}{|c|}{$16 \alpha-\mathrm{HSD}$} & \multicolumn{2}{|c|}{$16 \beta$-HSD } & \multirow{2}{*}{$\begin{array}{c}17 \alpha- \\
\mathrm{HSD} \\
17 \alpha-\mathrm{E}_{2}\end{array}$} & \multicolumn{2}{|c|}{$17 \beta$-HSD } & \multicolumn{2}{|c|}{$20 \alpha-H S D$} & \multirow{2}{*}{$\frac{20 \beta-H S D}{20 \beta-O H-}$} \\
\hline $\begin{array}{c}11 \beta- \\
\text { Andro- } \\
\text { stènediol }\end{array}$ & $\begin{array}{c}\text { Corti- } \\
\text { sol }\end{array}$ & $\begin{array}{c}16 \alpha-\mathrm{OH}- \\
\mathrm{E}_{1}\end{array}$ & $\mathrm{E}_{3}$ & $\underset{\substack{16 \\
E_{1}-\mathrm{OH}-}}{ }$ & Épi $E_{3}$ & & Testo & $17 \beta-E_{2}$ & $\begin{array}{c}20 \alpha-\mathrm{OH}- \\
\mathrm{Pg}\end{array}$ & $\begin{array}{c}\text { Pregna- } \\
\text { nediol }\end{array}$ & \\
\hline$(+)$ & $(+)$ & - & - & - & - & - & + & + & $(+)$ & $(+)$ & - \\
\hline- & $(+)$ & - & - & - & - & - & - & + & $(+)$ & $(+)$ & $(+)$ \\
\hline$(+)$ & $(+)$ & - & - & - & - & 一 & $(+)$ & $(+)$ & $(+)$ & + & $(+)$ \\
\hline$(+)$ & $(+)$ & - & - & - & - & - & $(+)$ & $(+)$ & $(+)$ & + & $(+)$ \\
\hline
\end{tabular}

AU 5

urénalien chez le Hérisson pendant la phase d'activité sexuelle

: faible; ++ : moyenne; +++ : forte

\begin{tabular}{|c|c|c|c|c|c|c|c|c|c|c|c|}
\hline \multicolumn{2}{|c|}{$11 \beta$-HSD } & \multicolumn{2}{|c|}{$16 \alpha-H S D$} & \multicolumn{2}{|c|}{$16 \beta-\mathrm{HSD}$} & $17 \alpha-$ & \multicolumn{2}{|c|}{$17 \beta-\mathrm{HSD}$} & \multicolumn{2}{|c|}{$20 \alpha$-HSD } & \multirow{2}{*}{$\frac{20 \beta-H S D}{\substack{20 \\
\mathrm{Pg}-\mathrm{OH}-}}$} \\
\hline $\begin{array}{c}11 \beta- \\
\text { Andro- } \\
\text { stènediol }\end{array}$ & $\begin{array}{c}\text { Corti- } \\
\text { sol }\end{array}$ & $\begin{array}{c}16 \alpha-\mathrm{OH}- \\
\mathrm{E}_{1}\end{array}$ & $E_{3}$ & $\begin{array}{c}16 \beta-\mathrm{OH}- \\
\mathrm{E}_{1}\end{array}$ & Épi $E_{3}$ & $17 \alpha-E_{2}$ & Testo & $17 \beta-E_{2}$ & $\begin{array}{c}20 \alpha-\mathrm{OH}- \\
\mathrm{Pg}\end{array}$ & $\begin{array}{c}\text { Pregna- } \\
\text { nediol }\end{array}$ & \\
\hline - & $(+)$ & - & 一 & - & - & - & - & + & $(+)$ & $(+)$ & $(+)$ \\
\hline - & $(+)$ & - & - & - & - & $(t)$ & - & $(+)$ & $(+)$ & - & - \\
\hline- & $t+$ & - & - & + & $(+)$ & $(t)$ & $(t)$ & $(+)$ & + & - & - \\
\hline
\end{tabular}


Cette homogénéité des types cellulaires est sans doute due à deux processus s'opérant de façon simultanée :

- une crois sance cytoplasmique et nucléaire des cellules interstitielles de type I ;

- une diminution quantitative des précurseurs stockés dans les cellules hypertrophiées de type 2 au profit de la libération d'un produit de synthèse.

\section{Hyperplasies interstitielles.}

Les ovaires de certaines femelles présentent des nodules de grande dimension (2 à $5 \mathrm{~mm}$ de diamètre). De forme grossièrement sphérique (fig. 4, p. II) ils sont surtout composés de cellules interstitielles hypertrophiées de types 2 et 3 . On ne les observe que chez des animaux très âgés dont les ovaires, parfois kystiques, sont pauvres en follicules.

Ces hyperplasies apparaissent chez $70 \mathrm{p}$. Ioo des vieux hérissons (âge $\geqslant 4$ ans environ). L'appréciation de l'âge se fait par examen de l'animal : aspect externe, état de la denture et coloration de l'utérus et du vagin (rosé chez les jeunes et les adultes, orangé chez les vieilles femelles).

Nous avons examiné 24 ovaires porteurs d'une telle hyperplasie, un vingt cinquième étant atteint d'une tumeur selon toute vraisemblance d'origine interstitielle.

En raison de l'importance de la population étudiée nous avons pu observer les différentes étapes du processus de tumorisation :

- Dans un premier temps le n. i. h. hyperplasié est remarquable par le nombre et l'hypertrophie des cellules interstitielles de type 2 ; il n'y a pas de mitose.

- Ensuite, certaines se transforment en cellules de type 3, en se lysant celles-ci entraînent 1'apparition de lacunes (fig. 5, pl. II). téristique.

- Le tissu se désorganise, les cellules n'ont plus leur forme polyédrique carac-

- La glande interstitielle perdrait ensuite son individualité et envahirait les autres tissus ovariens. A ce stade (I cas), on peut observer la présence de nombreux composés lipoprotéiques, probablement d'origine lytique (fig. 6, pl. II).

\section{F. - Analyse histoenzymologique}

Trente-neuf ovaires ont été soumis à 1'analyse histoenzymologique. Les résultats sont consignés dans le tableau 4.

Pour les enzymes prises en considération il n'y a pas chez le Hérisson de variation sensible d'activité enzymatique en fonction du cycle reproducteur annuel.

Deux enzymes sont nettement mises en évidence : la succino oxydase et la $\Delta_{5}-3 \beta$-HSD.

- L'activité succino déshydrogénase suggère l'existence d'un métabolisme élevé (égal à celui du corps jaune) au niveau du $n$. $\mathrm{i}$. h. pendant toutes les phases du cycle reproducteur annuel.

L'étude de cette enzyme confirme que les cellules interstitielles de type I sont bien inactives (fig. I, pl. III).

- La $\Delta_{5^{-}} 3 \beta$-HSD est abondante au sein du n. i. h. hyperplasié ou non, le dépôt de diformazan, fort important, y est sensiblement égal toute l'année (fig. 2 et 4 , p. III). (L'androstènediol et la D. H. E. A. permettent d'obtenir les meilleurs résultats). 
Pendant 1'anœstrus hivernal, le n. i. h., hyperplasié ou non, est le seul tissu ovarien présentant une forte activité $\Delta_{5^{-}} 3 \beta$-HSD.

D'autres HSD ont été mises en évidence dans les cellules de l'interstitielle hilaire ; les $3 \alpha-, 6 \beta$ - et I $7 \beta$-HSD sont après la $\Delta_{5^{-}} 3 \beta-H S D$, les enzymes les mieux représentées (fig. 3, pl. IV).

- Les équipements enzymatiques des différents types de corps jaune et du n. i. h. (hyperplasié ou non) ont été comparés (tabl. 5).

Les résultats indiquent qu'il n'existe pas de différence qualitative ou quantitative sensible entre les potentialités sécrétoires de ces deux tissus.

- Afin de mieux connaître la nature et l'origine du n. i. h. nous avons procédé à une analyse comparative des enzymes de la stéroïdogenèse et de la corticosurrénale et de l'interstitielle ovarienne (tab1. 5).

Le cortex surrénalien ne montre aucune activité $3 \alpha-\mathrm{HSD}$, ni $20 \beta-\mathrm{HSD}$, par contre, les $20 \alpha$ - et $6 \beta$-HSD y sont plus abondantes qu'au niveau du n. i. h. Avec le cortisol, le cortex développe une belle activité II $\beta$-HSD.

On ne peut donc pas assimiler le n. i. h. du Hérisson aux «Adréno Cortical Like cells " décrites par Duke (I952), Chester-Jones (I963) et Mossman (I965) dans le hile de l'ovaire d'autres Mammifères.

\section{III. - DISCUSSION}

Le n. i. h. apparaît chez la femelle impubère vers l'âge de 3 mois. Cette genèse peut être induite expérimentalement par des gonadotropines. Son apparition résulte donc de l'élévation gonadotrope prépubertaire.

Il n'est pas apparu de différence d'action entre $\mathrm{LH}$ et $\mathrm{FSH}$ mais l'expérimentation ayant été effectuée chez des animaux entiers, il est possible qu'un effet de synergie ait joué.

Les gonadotropines permettraient la différenciation des cellules de certains tubules du rete ovarii ou (et) de cellules conjonctives situées à leur contact, en cellules interstitielles, aboutissant ainsi à la formation du n. i. h.

Nous avons montré que les cellules interstitielles (type 2) de l'ovaire du Hérisson sont soumises à des variations morphologiques cycliques et qu'elles synthétisent des stéroïdes.

Cette évolution est analogue à ce qui est observé au niveau de la glande interstitielle des autres espèces. Chez ces dernières, les cellules interstitielles sont toujours hypertrophiées avant ou au moment de l'ovulation; elles marquent un net fléchissement peu de temps après (Herlant, I956 ; Mossman, I964; KoEring, I969).

Comme la glande interstitielle des autres Mammifères, le n. i. h. pourrait sécréter un stéroïde ayant un rôle (inconnt1) au moment de l'ovulation.

Il est délicat de tirer une conclusion ferme sur 1'activité stéroïdogène après la seule analyse histoenzymologique, le défaut principal de cette technique étant la fuite de certaines enzymes solubles dans le milieu d'incubation. Cette solubilité varie suivant la localisation intracellulaire de l'enzyme, le tissu et parfois même l'espèce animale. 
La $20 \alpha$-HSD est réputée soluble chez la Lapine où son activité est forte au sein de l'interstitielle ovarienne (HILLIARD et al., I967, I969). Chez le Rat, BALOGH (I964) ne la révèle que difficilement alors que chez la Taupe, Gougeon (1972) met en évidence, à l'aide de cette technique histoenzymologique, non seulement son abondance, mais des variations cycliques importantes de son niveau d'activité.

Chez le Hérisson, cette méthode nous permet de faire quelques remarques :

- Il est intéressant de noter la similitude existant entre les équipements enzymatiques du n. i. h. et du corps jaune.

- Comme de plus, la $\Delta_{5}-3 \beta$-HSD est l'enzyme la plus active, une synthèse de progestérone, par le $n$. i. h. peut être envisagée.

Cependant, seule une étude plus approfondie permettra de vérifier cette hypothèse déjà démontrée chez le Rat (Uchida et al., I969; Armstrong et al., I969), le Macaque (Koering, I969), le Hamster (LukASZEWSKa et GreENWALD, I970).

Le n. i. h. présente toujours, quelle que soit la phase du cycle reproducteur annuel, une stéroïdogenèse et un métabolisme général importants. Cette activité reflète-t-elle l'existence d'une sécrétion continue? Les cellules interstitielles sont hypertrophiées et bourrées de graisses dans deux cas : pendant l'anœestrus hivernal et quand le n. i. h. est hyperplasié.

Bien que la coloration par le noir Soudan B et le test de Schultz soient négatifs, ces lipides peuvent être composés de cholestérol, car de nombreuses glandes endocrines stéroïdogènes contiennent des réserves de cholestérol lié, histochimiquement impossible à mettre en évidence (Guraya, I973).

Les cellules interstitielles hypertrophiées ne synthétiseraient pas de stéroïde biologiquement actif, mais stockeraient du cholestérol lié, mobilisable pour une stéroïdogenèse ultérieure. Certaines observations confirment l'inactivité sécrétoire de ce type cellulaire, notamment l'absence de différence histologique significative entre les effecteurs sexuels des animaux, que le $\mathrm{n}$. i. h. soit hyperplasié ou non.

- Ces cellules interstitielles hypertrophiées se rencontrent pendant l'anœstrus hivernal et au niveau des $n$. i. h. hyperplasiés (donc chez de vieux animaux). Dans ces deux cas LH est peu ou pas libérée. Or cette hormone favorise la synthèse de la progestérone à partir du cholestérol estérifié, en activant la cholestérol-estérase (ARMSTRONG et al., I969). Sa libération aurait pour résultat de mobiliser le cholestérol lié, provoquant ainsi une réduction des graisses stockées donc de la taille des cellules.

Comment interpréter la mise en évidence d'une forte activité $\Delta_{5}-3 \beta$-HSD au niveau des cellules interstitielles hypertrophiées?

Ces cellules ne sécréteraient pas en raison du freinage s'exerçant au niveau de la cholestérol-estérase. Cependant, la stéroïdogenèse peut normalement se poursuivre si on introduit dans le milieu d'incubation le stéroïde dont la synthèse est bloquée expliquant ainsi l'observation d'un dépôt de diformazan.

Ultérieurement, l'injection de LH à des animaux en anœstrus hivernal ou porteurs d'un $\mathrm{n}$. i. h. hyperplasié permettrait de vérifier si cette hormone favorisant la mobilisation du cholestérol, entraîne une diminution de volume des cellules interstitielles hypertrophiées. De plus, la nature du stéroïde synthétisé pourrait être éclaircie par l'incubation, avec et sans $\mathrm{LH}$, de nodules hyperplasiés en présence de précurseurs marqués. 


\title{
REMERCIEMENTS
}

Nous tenons à remercier le professeur CANIVENC de nous avoir accueilli au Centre d'Études biologiques des Animaux sauvages du C. N. R. S., le professeur PEYRE au Laboratoire duquel le présent travail a été effectué et le professeur Thibaulr des conseils et de l'aide matérielle qu'il nous a apportés.

\author{
SUMMARY \\ ORIGINAL, ASPEC'TS OF THE OVARIAN INTERSTITIAL GLAND \\ IN THE HEDGEHOG : MORPHOLOGY, HISTOGENESIS, HYPERPLASIA, \\ STEROIDOGENIC ENZYMES
}

The ovarian interstitial gland in the European hedgehog is in the form of a nodule located at the hilum; it is called the hilar interstitial nodule (n. i. h.).

Situated near the rete ovarii tubules, it only appears in the young female at 3 months $(6$ to 9 months before puberty), but may be induced earlier by LH or (and) FSH.

It results from the evolution of certain rete ovarii tubule cells and its volume increases progressively due to the contribution and differentiation of spindle-shaped connective cells from the ovarian medulla.

Histoenzymologic analysis shows that $n$. i. h. steroidogenic enzymes resemble those of the corpus luteum. $\Delta_{5}-3 \beta-\mathrm{HSD}$ is the most active enzyme, and I $\beta$ - and $6 \beta$-HSD are also determined.

Decrease in interstitial cell volume during the period of sexual activity expresses hormone synthesis followed by secretion.

The $\mathrm{n}$. i. h. is subject to hyperplasia in old animals $(\geqslant 4$ years). The nodule then becomes large $(2-5 \mathrm{~mm}$ in diameter). Hypertrophied interstitial cells constitute the only cell type and there is no mitosis.

\section{RÉFÉRENCES BIBLIOGRAPHIQUES}

Armstrong D. T., Miller L. S., Knudsen K. A., ig69. Regulation of metabolism and progesterone production in Rat corpora lutea and ovarian interstitial tissue by prolactin and luteinizing hormone. Endocrinology, 85, 394-401.

Aschнег P., I968. Contenu hypophysaire en hormone lutéinisante (LH) et réaction histophysiologique à la LH circulante du tissu interstitiel ovarien chez divers types de Rattes séniles. C. R. Acad. Sci., 267, $1397-1400$.

Athias M., 1923. Sur la signification des phénomènes d'atrésie folliculaire et des cellules interstitielles de l'ovaire. C. R. Séanc. Soc, Biol., 88, $1315-1318$.

BaLboni G. C., I969. Observations on the ovary of the Hedgehog (Erinaceus europeus) in physiological and experimental hibernation and awakening. Acta Anat., suppl., 58, 60-7r.

Baillie A. H., Ferguson M. M., McHart D., I966. Development in steroid histochemistry, 186 p. Acad. Press, London and N. Y.

BALOGH K, I 964 . A histochemical method for the demonstration of $20 \alpha$-hydroxysteroid dehydrogenase activity in rat ovary. J. Histochem. Cytochem., 12, 670-673.

Chester-Jones I., Henderson I. W., 1963. The ovary of the I3-lined ground squirrel (Citellus tridecemlineatus MIтсHELL) after adrenalectomy. J. Endocr., 26, 265-272.

Deanesly R., I934. The reproductive processes of certain mammals. Part IV : The reproductive cycle, of the female Hedgehog. Ph. Trans. $r$. Soc. London, $233 \mathrm{~B}, 239-276$.

Duke K. L., I952. Ovarian histology of Ochotona princeps : the rocky mountain pika. Anat. Rec. 112, $737-759$.

Girod C., Dubois P., Cure M., 1967. Recherches sur les corrélations hypophysogénitales chez la femelle de Hérisson (Erinaceus europeus L.). Ann. Endocrinol., 28, 581-6ro.

Gougeon A., 1972. Analyse histologique et histoenzymologique de l'interstitielle ovarienne chez deux Insectivores européens: la Taupe (Talpa europea) et le Hérisson (Erinaceus europeus). Thèse de $3^{\text {e }}$ cycle, Physiologie animale, Paris, i 2 p. 
Guraya S. S., I973. Interstitial gland tissue of mammalian ovary. Acta Endocr. Copenh., 72, suppl. 171, 5-27.

Hilliard J., Penardi R., Sawyer C. H., I967. A functional role for $20 \alpha$-hydroxy pregn-4-en-3-one in the Rabbit. Endocrinology, 80, $901-909$.

KonRing M. J., I969. Cyclic changes in ovarian morphology during the menstrual cycle in Macaca mulatta, Amer, J. Anat., 126, 73-ror.

Levy H., Deane H. W., Rubin B. L., I959. Visualisation of steroid-3 $\beta$-ol-dehydrogenase activity in tissue of intact and hypophysectomised rats. Endocrinology, 65, 932-942.

Limon M., I902-I903. Étude histologique et histogénique de la glande interstitielle de l'ovaire. Arch. Anat. micr. Morphol. expér., 5, I55-188.

Lukaszewska J. H., Greenwald G.S., 1970. Progesterone levels in the cyclic and pregnant Hamster. Endocrinology, 86, I-9.

Marchal-Dacheux F., Peyre A., I97o. Variations saisonnières des hydroxystéroïdo-déshydrogénases $\left(A_{5}-3 \beta\right.$ et $\left.17 \beta\right)$ dans l'ovaire de Hérisson : Erinaceus europeus, C. R. Soc. Biol, 164, 2084.

Mossman H. W., I966. The rodent ovary. In : Comparative biology of reproduction in mammals, RowLands I. W. ed., Acad. Press London and N. Y., 455-470.

Nachlas M. M., Tsou K. C., Desouza E., Cheng C. S., Seligman A. M., I957. Cytochemical demonstration of succinic dehydrogenase by the use of a new p. nitro phenyl substitued ditetrazole. J. Histochem. Cytochem., 5, 420-436.

Ponse K., 1966. Action de LH sur l'ovaire. In : Fonction endocriniennes de l'ovaire, JAYLE F. ed., Gauthiers-Villars, Paris, I7-52.

Solari A., I973. Étude quantitative d'organes et de tissus. I. Méthode d'estimation des volumes. Ann. Biol. anim. Bioch. Biophys., 13, 247-265.

Uchida K., Kadowaki M., MiYake T., ig69. Ovarian secretion of progesterone and 20 -hydroxypregn-4-en-3-one during rat estrous cycle in chronological relation to pituitary release of luteinizing hormone. Endocr. Jap., 16, 227-237.

Velloso de Pinho A., I923. Atrésie de l'épithélium folliculaire ovarique chez les Mammifères. C. $R$. Soc. Biol., 88, $830-833$.

Walin T., Solvio A., Kristofferson R., I968. Histological changes in the reproductive system of female Hedgehogs during the hibernation season. Ann. Zool. Fenn., 5, 227-229.

Wattenberg L. W., I958. Microscopic histochemical demonstration of steroid 3-o $\beta$-dehydrogenase in tissue sections. J. Histochem. Cytochem., 6, 225-232.

\section{PLANCHE I}

FIG. I

N. i. h. enclavé dans un conjonctif lâche chez une femelle en reprise d'activité génitale (printemps). Les cellules interstitielles ont toutes la même taille ( $X$ I6o).

FIG. 2

Proximité des tubules du rete ovarii (r. o.) et du n. i. h. $(\times 250)$.

FIG. 3

N. i. h. jeune ; sa taille et sa forme sont semblables à celles des tubules du rete ovarii (r. o.) dont il semble dériver ( $X$ IOO).

FIG. 4

Transformation de cellules des tubules du rete ovarii (r. o.) en cellules intertitielles. Le $n$. i. h. renferme des cellules interstitielles de type I et de type $2(\times 250)$. 

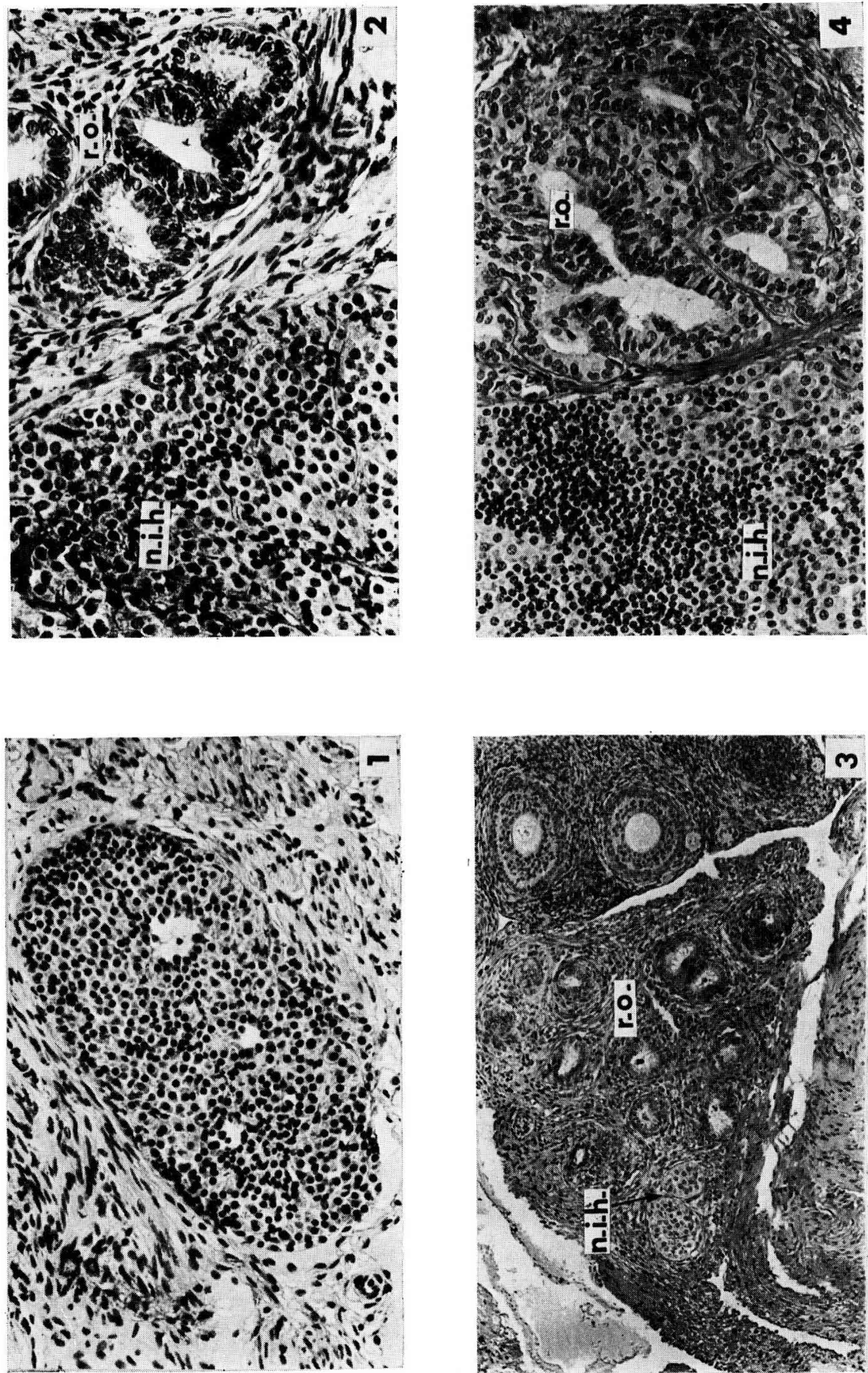


\section{PLANCHE II}

FIG. 1

Cellules interstitielles inactives de type 1. ( $\times$ 1000)

FIG. 2

Cellules interstitielles de type $2 .(\times 1000)$

FIG. 3

Cellules interstitielles de type 2 (hypertrophiées) et de type 3 (hyperplasie interstitielle). ( $\times$ 1000)

FIG. 4

Hyperplasie interstitielle. $(\times 25)$

FIG. 5

Evolution lytique de cellules interstitielles de type 3 entraînant l'apparition de lacunes. $(\times 250)$

FIG. 6

Tumeur ayant pour origine un n. i. h. hyperplasié ; la plupart des cellules dégénèrent. $(\times 1000)$ 

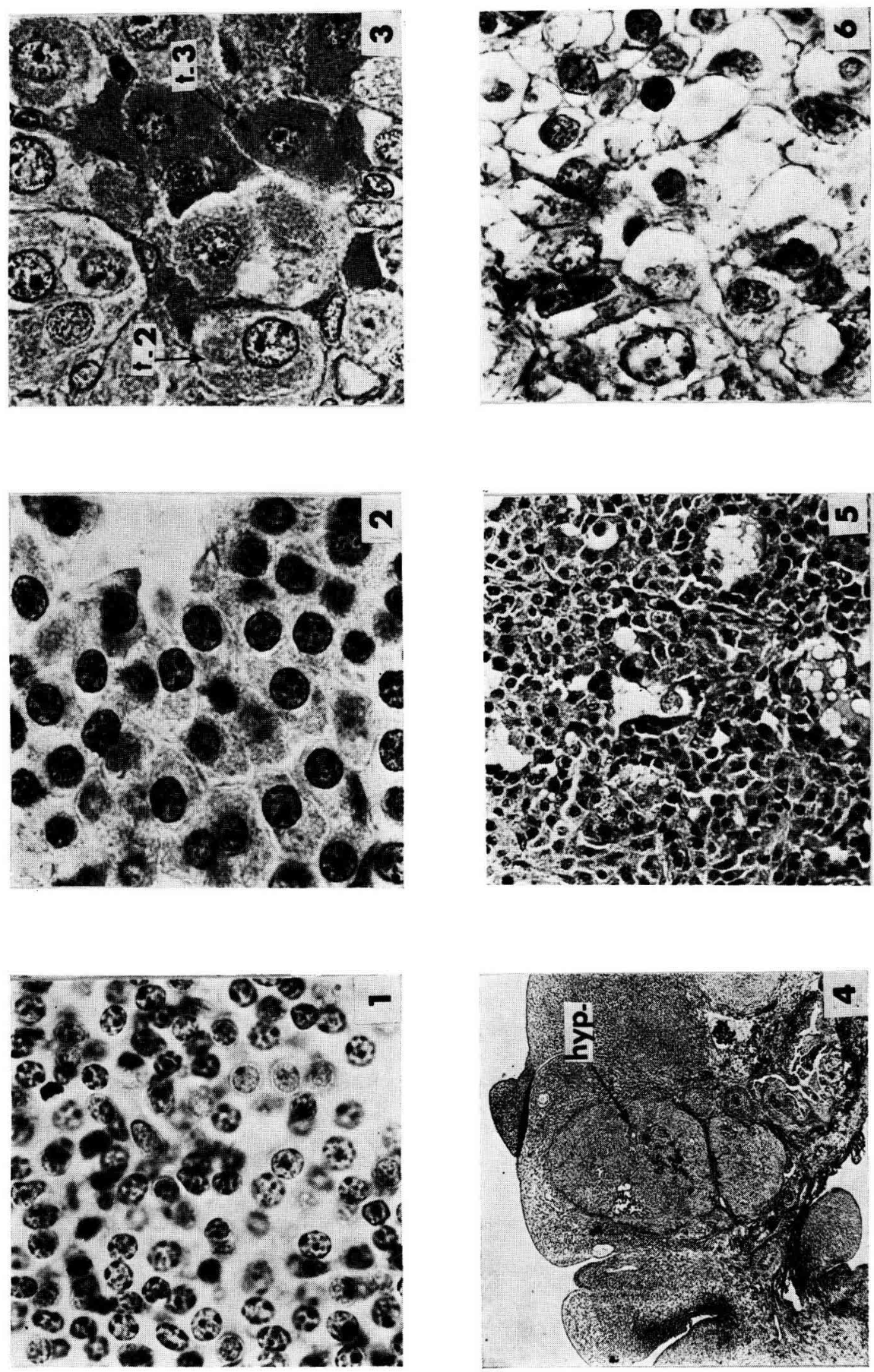


\section{PLANCHE III}

Fig. 1

Succino déshydrogénase, anœstrus hivernal; les cellules de type 1 (C. 1 ) sont moins actives que les cellules de type 2 (C. 2 ). $(\times 100)$

FIG. 2

Forte activité $\triangle_{5}-3 \beta$-HSD (substrat: D. H. E. A.) au sein du n. i. h. pendant la reprise d'activité génitale. $(\times 100)$

FIG. 3

Faible activité $17 \beta$-HSD (substrat : œstradiol-17 $\beta$ ) au sein du n. i. h. pendant la reprise d'activité génitale. $(\times 160)$

FIG. 4

n. i. h. hyperplasié chez une femelle gestante. (Activité $\left.\Delta_{5}-3 \beta-H S D\right) .(\times 10)$ 

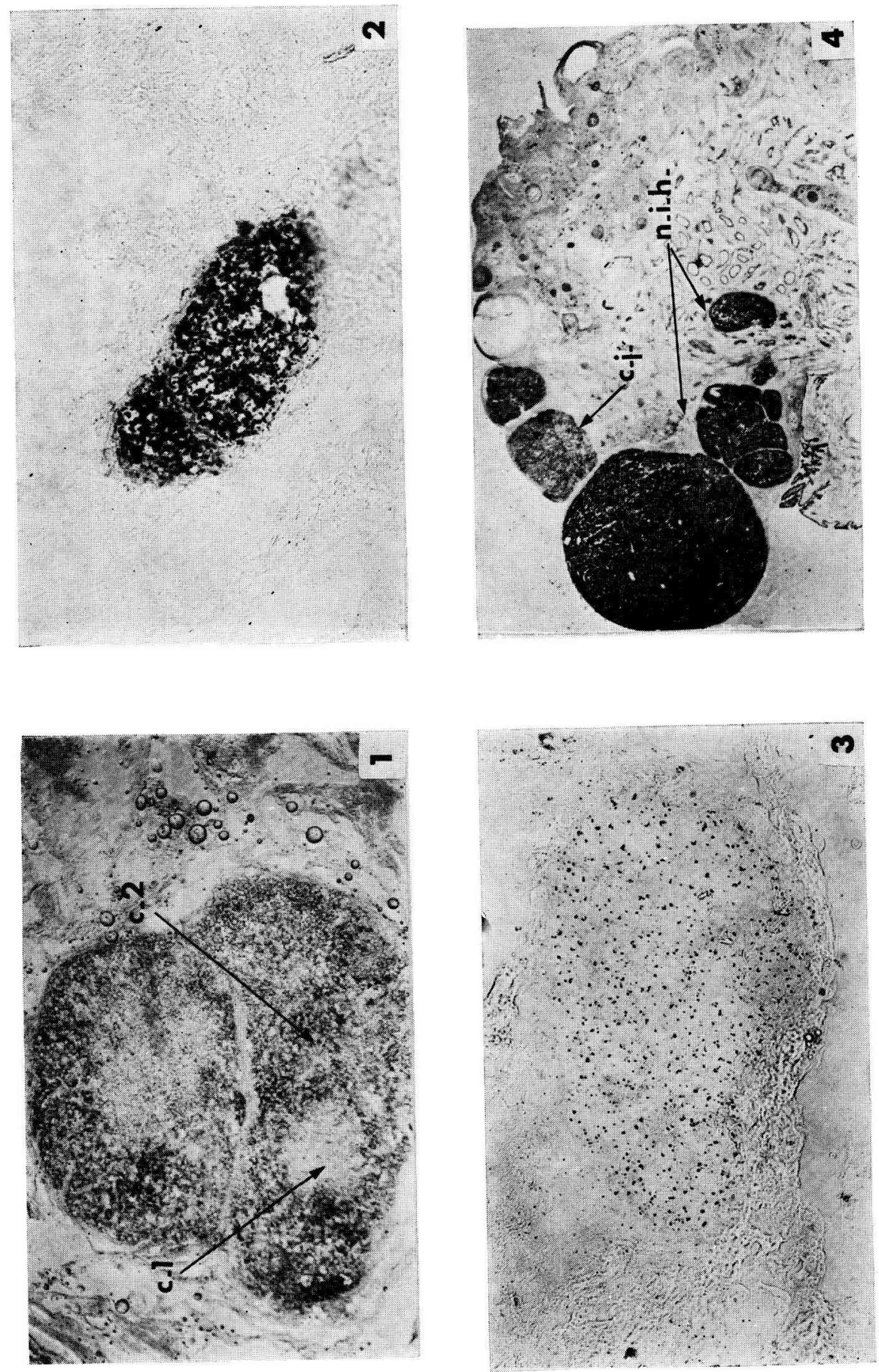